\title{
Blow-Up Phenomena for a Class of Parabolic Systems with Time Dependent Coefficients
}

\author{
Lawrence E. Payne ${ }^{1}$, Gérard A. Philippin ${ }^{2}$ \\ ${ }^{1}$ Department of Mathematics, Cornell University, Ithaca, USA \\ ${ }^{2}$ Département de Mathématiques et de Statistique, Université Laval, Québec City, Canada \\ Email: gphilip@mat.ulaval.ca
}

Received February 8, 2012; revised March 7, 2012; accepted March 14, 2012

\begin{abstract}
Blow-up phenomena for solutions of some nonlinear parabolic systems with time dependent coefficients are investigated. Both lower and upper bounds for the blow-up time are derived when blow-up occurs.
\end{abstract}

Keywords: Parabolic Systems; Blow-Up; Sobolev Type Inequality

\section{Introduction}

It is well known that the solutions of parabolic problems may remain bounded for all time, or may blow-up in finite or infinite time. When blow-up occurs at time $t^{*}$, the evaluation of $t^{*}$ is of great practical interest.

In a recent paper [1] Payne and Schaefer have investigated the blow-up phenomena of solutions in some parabolic systems of equations under homogeneous Dirichlet boundary conditions. The contribution of this note is to extend their investigations to a class of parabolic systems with time dependent coefficients. The case of a single parabolic equation was investigated recently in [2].

There is an abounding literature dealing with blow-up phenomena of solutions to parabolic partial differential equations. We refer the interested readers to [3-5]. A variety of physical, chemical, biological applications are discussed in $[5,6]$. Further references to the field are [1,7-19]. In this note we investigate the blow-up phenomena of the solution $(u, v)$ of the following parabolic system

$$
\left\{\begin{array}{l}
u_{t}=\Delta u+k_{1}(t) f_{1}(v), x=\left(x_{1}, \cdots, x_{N}\right) \in \Omega, t \in\left(0, t^{*}\right) \\
v_{t}=\Delta v+k_{2}(t) f_{2}(u), x \in \Omega, t \in\left(0, t^{*}\right) \\
u(x, t)=v(x, t)=0, x \in \partial \Omega, t \in\left(0, t^{*}\right) \\
u(x, 0)=u_{0}(x) \geq 0, v(x, 0)=v_{0}(x) \geq 0, x \in \Omega
\end{array}\right.
$$

where $\Omega$ is a bounded domain in $\mathbb{R}^{N}, N \geq 2$. The initial data $\left(u_{0}, v_{0}\right)$ as well as the data $k_{1}(t), k_{2}(t)$, $f_{1}(t), f_{2}(t)$ are assumed nonnegative, so that the solution $(u, v)$ of (1.1) will be nonnegative by the maximum principle. More specific assumptions on the data will be made later.
In Section 2 we derive conditions on the data of problem (1.1) sufficient to guarantee that blow-up will occur, and derive under these conditions some upper bound for $t^{*}$. In Section 3 we derive some lower bounds for the blow-up time $t^{*}$ when blow-up occurs. However this section is limited to the case of $\Omega$ in $\mathbb{R}^{2}$ and in $\mathbb{R}^{3}$ respectively, because our technique makes use of some Sobolev type inequalities available in $\mathbb{R}^{2}$ and in $\mathbb{R}^{3}$ only. For convenience we include the proof of one of these inequalities in Section 4.

\section{Conditions for Blow-Up in Finite Time $t^{*}$}

Let $\lambda_{1}$ be the first eigenvalue and $\phi_{1}$ be the associated eigenfunction of the Dirichlet-Laplace operator defined as

$$
\begin{gathered}
\Delta \phi_{1}+\lambda_{1} \phi_{1}=0, \phi_{1}>0, x \in \Omega ; \phi_{1}=0, x \in \partial \Omega, \\
\int_{\Omega} \phi_{1} \mathrm{~d} x=1 .
\end{gathered}
$$

Let the auxiliary function $\theta(t)$ be defined in $\left(0, t^{*}\right)$ as

$$
\theta(t):=\psi(t)+\chi(t)
$$

with

$$
\psi(t):=\int_{\Omega} u \phi_{1} \mathrm{~d} x, \quad \chi(t):=\int_{\Omega} v \phi_{1} \mathrm{~d} x,
$$

where $(u, v)$ is the solution of problem (1.1). We assume in this section that $\Omega$ is a bounded domain of $\mathbb{R}^{N}, N \geq 2$, and that

$$
\begin{aligned}
& f_{1}(s) \geq s^{p}, p=\text { constant }>1, \\
& f_{2}(s) \geq s^{q}, q=\text { constant }>1, s>0, \\
& \min _{t>0}\left\{k_{1}(t), k_{2}(t)\right\}=: K>0 .
\end{aligned}
$$


We then compute

$$
\begin{aligned}
\psi^{\prime}(t) & =\int_{\Omega}\left[\Delta u+k_{1} f_{1}(v)\right] \phi_{1} \mathrm{~d} x \\
& \geq-\lambda_{1} \psi(t)+k_{1}(t) \int_{\Omega} v^{p} \phi_{1} \mathrm{~d} x
\end{aligned}
$$

Making use of Hölder's inequality, we have

$$
\chi(t)=\int_{\Omega} v \phi_{1} \mathrm{~d} x \leq\left(\int_{\Omega} v^{p} \phi_{1} \mathrm{~d} x\right)^{\frac{1}{p}} .
$$

Combining (2.7) and (2.8), we obtain

$$
\psi^{\prime}(t) \geq-\lambda_{1} \psi(t)+k_{1}(t)(\chi(t))^{p} .
$$

A similar computation leads to

$$
\chi^{\prime}(t) \geq-\lambda_{1} \chi(t)+k_{2}(t)(\psi(t))^{q} .
$$

Adding (2.9) and (2.10), we obtain

$$
\theta^{\prime}(t)=\psi^{\prime}(t)+\chi^{\prime}(t) \geq-\lambda_{1} \theta(t)+K\left(\psi^{q}+\chi^{p}\right),
$$

where $K$ is defined in (2.6). We first investigate the particular case $p=q$. Making use of Hölder's inequality, we have

$$
\psi^{q}+\chi^{q} \geq 2^{1-q}(\psi+\chi)^{q}=2^{1-q}(\theta(t))^{q} .
$$

Inserted in (2.11), we obtain the first order differential inequality

$$
\theta^{\prime}(t) \geq-\lambda_{1} \theta+2^{1-q} K \theta^{q}, t \in\left(0, t^{*}\right) .
$$

Integrating (2.13) from 0 to $t$, we obtain the inequality

$$
\begin{aligned}
(\theta(t))^{1-q} & \leq e^{(q-1) \lambda_{1} t}\left\{(\theta(0))^{1-q}-\frac{2^{1-q} K}{\lambda_{1}}\right\}+\frac{2^{1-q} K}{\lambda_{1}} \\
& =: \varepsilon(t) .
\end{aligned}
$$

Suppose that the data satisfy the condition

$$
\theta(0)>2\left(\frac{\lambda_{1}}{K}\right)^{1 /(q-1)} .
$$

Then $\varepsilon(t)$ vanishes at some time $t_{0}>0$, and $\theta(t)$ must blow up at some time $t^{*} \leq t_{0}$. We obtain

$$
t^{*} \leq t_{0}:=-\frac{1}{(q-1) \lambda_{1}} \log \left\{1-\frac{2^{q-1} \lambda_{1}}{K(\theta(0))^{q-1}}\right\} .
$$

In the general case, we suppose without loss of generality that $p>q$, and make use of the inequality

$$
\chi^{q}=\left(c \chi^{p}\right)^{\frac{q}{p}}\left(c^{-\frac{q}{p-q}}\right)^{\frac{p-q}{p}} \leq \frac{q}{p} c \chi^{p}+\frac{p-q}{p} c^{-\frac{q}{p-q}},
$$

valid for arbitrary $c>0$. Choosing $c:=\frac{p}{q}$, we obtain

$$
\chi^{q} \leq \chi^{p}+Q
$$

with

$$
Q:=\frac{p-q}{p}\left(\frac{q}{p}\right)^{\frac{q}{p-q}}>0 .
$$

Inserted in (2.12), we obtain the first order differential inequality

$$
\theta^{\prime}(t) \geq 2^{1-q} K \theta^{q}-\lambda_{1} \theta-K Q=: \Theta(\theta) .
$$

Suppose that the initial data are so large that $\Theta(\theta(0))>0$. Then $\theta(t)$ is increasing for $t$ small. Since $\Theta(\theta)$ is increasing in $\theta$ from its negative minimum, it follows then that $\Theta(\theta(t))$ is increasing for $t>0$. This shows that $\theta^{\prime}(t)$ remains positive, so that $\theta(t)$ blows up at time $t^{*}$. Integrating (2.20) leads to the following upper bound for $t^{*}$

$$
t^{*}=\int_{0}^{t^{*}} \mathrm{~d} t \leq \int_{\theta(0)}^{\infty} \frac{\mathrm{d} \theta}{\Theta(\theta)} .
$$

These results are summarized in the following.

\section{Theorem 1}

1) Assume (2.5) with $p=q>1$, (2.6), and (2.15). Then $\theta(t)$ defined in (2.3) blows up at finite time $t^{*}$ bounded above by (2.16).

2) Assume (2.5) with $p>q>1$, (2.6), and $\Theta(\theta(0))>0$ with $\Theta(\theta)$ defined in (2.20). Then $\theta(t)$ blows up at finite time $t^{*}$ bounded above by (2.21).

To conclude this section, we note that if the condition (2.6) is replaced by

$$
\min _{t>\tau}\left\{k_{1}(t), k_{2}(t)\right\}=: K>0,
$$

then we have to replace the initial data $\theta(0)$ by $\theta(\tau)$ in Theorem 1. Clearly we may use a lower bound for $\theta(\tau)$. For instance we may integrate the differential inequality

$$
\theta^{\prime} \geq-\lambda_{1} \theta
$$

that follows from (2.11), leading to the lower bound

$$
\theta(\tau) \geq e^{-\lambda_{1} \tau} \theta(0) .
$$

\section{Lower Bounds for $t^{*}$}

In this section we assume that the data $f_{1}, f_{2}$, satisfy the conditions

$$
0 \leq f_{1}(s) \leq s^{p}, p>1 ; 0 \leq f_{2}(s) \leq s^{q}, q>1, s>0,
$$

and that the data $k_{1}(t), k_{2}(t)$ are nonnegative for all $t>0$. Moreover the solution is assumed to blow up in the sense that $\Phi(t) \rightarrow \infty$ as $t \rightarrow t^{*}$, where $\Phi(t)$ is defined as

$$
\Phi(t):=M_{1}^{-1} U(t)+M_{2}^{-1} V(t),
$$


with

$$
\begin{aligned}
& U(t):=\int_{\Omega} u^{2 q} \mathrm{~d} x, \quad M_{1}:=\int_{\Omega} u_{0}^{2 q} \mathrm{~d} x, \\
& V(t):=\int_{\Omega} v^{2 p} \mathrm{~d} x, \quad M_{2}:=\int_{\Omega} v_{0}^{2 p} \mathrm{~d} x .
\end{aligned}
$$

Differentiating (3.3) and making use of (1.1), (3.1), we obtain

$$
\begin{aligned}
U^{\prime}(t) & \leq 2 q \int_{\Omega} u^{2 q-1}\left[\Delta u+k_{1}(t) v^{p}\right] \mathrm{d} x \\
& =2 q k_{1}(t) \int_{\Omega} u^{2 q-1} v^{p} \mathrm{~d} x-2 q(2 q-1) J(t),
\end{aligned}
$$

with

$$
J(t):=\int_{\Omega} u^{2(q-1)}|\nabla u|^{2} \mathrm{~d} x .
$$

Making use of Schwarz and Hölder's inequalities we have

$$
\begin{aligned}
& \int_{\Omega} u^{2 q-1} v^{p} \mathrm{~d} x \leq\left(\int_{\Omega} u^{2(2 q-1)} \mathrm{d} x \int_{\Omega} v^{2 p} \mathrm{~d} x\right)^{1 / 2} \\
& \leq\left(\int_{\Omega} u^{4 q} \mathrm{~d} x\right)^{\frac{q-1}{2 q}}\left(\int_{\Omega} u^{2 q} \mathrm{~d} x\right)^{1 / 2 q}\left(\int_{\Omega} v^{2 p} \mathrm{~d} x\right)^{1 / 2} .
\end{aligned}
$$

In $\mathbb{R}^{2}$ we make use of the following Sobolev type inequality

$$
\int_{\Omega} u^{4 q} \mathrm{~d} x \leq \frac{q^{2}}{2} \int_{\Omega} u^{2(q-1)}|\nabla u|^{2} \mathrm{~d} x \int_{\Omega} u^{2 q} \mathrm{~d} x,
$$

derived in the last section of the paper. Combining (3.7) and (3.8), we obtain

$$
\begin{aligned}
& \int_{\Omega} u^{2 q-1} v^{p} \mathrm{~d} x \\
& \leq\left(\frac{q^{2}}{2}\right)^{\frac{q-1}{2 q}}(J(t))^{\frac{q-1}{2 q}}\left(\int_{\Omega} u^{2 q} \mathrm{~d} x\right)^{1 / 2}\left(\int_{\Omega} v^{2 p} \mathrm{~d} x\right)^{1 / 2} \\
& \leq \frac{1}{2}\left(\frac{q^{2}}{2}\right)^{\frac{q-1}{2 q}}(J(t))^{\frac{q-1}{2 q}} M_{1}^{1 / 2} M_{2}^{1 / 2} \Phi(t),
\end{aligned}
$$

where we have used the arithmetic-geometric mean inequality. Making use of the inequality

$$
\begin{aligned}
& a^{r} b^{1-r} \leq r a+(1-r) b, \\
& r \in(0,1), a>0, b>0,
\end{aligned}
$$

we have

$$
\begin{aligned}
(J(t))^{\frac{q-1}{2 q}} \Phi & =\left(c^{-1} J\right)^{\frac{q-1}{2 q}}\left(c^{\frac{q-1}{q+1}} \Phi^{\frac{2 q}{q+1}}\right)^{\frac{q+1}{2 q}} \\
& \leq \frac{q-1}{2 q} c^{-1} J(t)+\frac{q+1}{2 q} c^{\frac{q-1}{q+1}} \Phi^{\frac{2 q}{q+1}},
\end{aligned}
$$

valid for arbitrary $c>0$ to be chosen later. Inserted in (3.9) and (3.5), we obtain

$$
\begin{aligned}
& U^{\prime}(t) \\
& \leq\left\{\frac{(q-1) k_{1}(t)}{2}\left(\frac{q^{2}}{2}\right)^{\frac{q-1}{2 q}} M_{1}^{1 / 2} M_{2}^{1 / 2} c^{-1}-2 q(2 q-1)\right\} J(t) \\
& +\frac{(q+1) k_{1}(t)}{2}\left(\frac{q^{2}}{2}\right)^{\frac{q-1}{2 q}} M_{1}^{1 / 2} M_{2}^{1 / 2} c^{\frac{q-1}{q+1}} \Phi^{\frac{2 q}{q+1}} .
\end{aligned}
$$

We now select

$$
c:=\frac{(q-1) k_{1}(t)}{4 q(2 q-1)} M_{1}^{1 / 2} M_{2}^{1 / 2}\left(\frac{q^{2}}{2}\right)^{\frac{q-1}{2 q}}
$$

in order to have \{\}$=0$ in (3.12), arriving at

$$
M_{1}^{-1} U^{\prime}(t) \leq F(q) M_{1}^{-\frac{1}{q+1}} M_{2}^{\frac{q}{q+1}}\left(k_{1}(t)\right)^{\frac{2 q}{q+1}}(\Phi(t))^{\frac{2 q}{q+1}},
$$

with

$$
F(q):=2^{-\frac{2(2 q-1)}{q+1}}(q+1)\left(\frac{q(q-1)}{2 q-1}\right)^{\frac{q-1}{q+1}}
$$

A similar computation leads to

$$
\begin{aligned}
& M_{2}^{-1} V^{\prime}(t) \\
& \leq F(p) M_{1}^{\frac{p}{p+1}} M_{2}^{-\frac{1}{p+1}}\left(k_{2}(t)\right)^{\frac{2 p}{p+1}}(\Phi(t))^{\frac{2 p}{p+1}},
\end{aligned}
$$

where $V(t)$ is defined in (3.4). In $\mathbb{R}^{3}$, we replace (3.7) by

$$
\begin{aligned}
\int_{\Omega} u^{2 q-1} v^{p} \mathrm{~d} x & \leq\left(\int_{\Omega} u^{2(2 q-1)} \mathrm{d} x \int_{\Omega} v^{2 p} \mathrm{~d} x\right)^{1 / 2} \\
& \leq\left(\int_{\Omega} u^{6 q} \mathrm{~d} x\right)^{\frac{q-1}{4 q}}\left(\int_{\Omega} u^{2 q} \mathrm{~d} x\right)^{\frac{q+1}{4 q}}\left(\int_{\Omega} v^{2 p} \mathrm{~d} x\right)^{1 / 2} .
\end{aligned}
$$

and make use of the Sobolev type inequality

$$
\begin{aligned}
\left(\int_{\Omega} u^{6 q} \mathrm{~d} x\right)^{1 / 6} & \leq \gamma q\left(\int_{\Omega} u^{2(q-1)}|\nabla u|^{2} \mathrm{~d} x\right)^{1 / 2} \\
& =\gamma q(J(t))^{1 / 2}
\end{aligned}
$$

derived by Talenti in [20] with $\gamma:=4^{1 / 3} 3^{-1 / 2} \pi^{-2 / 3}$. Inserted in (3.17), we obtain

$$
\begin{aligned}
& \int_{\Omega} u^{2 q-1} v^{p} \mathrm{~d} x \\
& \leq C(q)(J(t))^{\frac{3(q-1)}{4 q}}\left(M_{1}^{-1} U(t)\right)^{\frac{q+1}{4 q}}\left(M_{2}^{-1} V(t)\right)^{1 / 2} M_{1}^{\frac{q+1}{4 q}} M_{2}^{1 / 2},
\end{aligned}
$$

with

$$
C(q):=(\gamma q)^{\frac{3(q-1)}{2 q}} .
$$

Moreover we make use of (3.10) to write 


$$
\begin{aligned}
& \left(M_{1}^{-1} U(t)\right)^{\frac{q+1}{4 q}} J^{\frac{3(q-1)}{4 q}} \\
= & \left(c^{-1} J\right)^{\frac{3(q-1)}{4 q}}\left[c^{\frac{3(q-1)}{q+3}}\left(M_{1}^{-1} U\right)^{\frac{q+1}{q+3}}\right]^{\frac{q+3}{4 q}} \\
\leq & \frac{3(q-1)}{4 q} c^{-1} J+\frac{q+3}{4 q} c^{\frac{3(q-1)}{4 q}}\left(M_{1}^{-1} U\right)^{\frac{q+1}{q+3}},
\end{aligned}
$$

with arbitrary $c>0$ to be chosen later. Combining (3.5), (3.19) and (3.21), we obtain

$$
\begin{aligned}
& U^{\prime}(t) \\
& \leq\left\{\frac{3(q-1)}{2} C(q) M_{1}^{\frac{q+1}{4 q}} M_{2}^{1 / 2}\left(M_{2}^{-1} V\right)^{1 / 2} k_{1}(t) c^{-1}\right. \\
& -2 q(2 q-1)\} J(t) \\
& +\frac{q+3}{2} C(q) M_{1}^{\frac{q+1}{4 q}} M_{2}^{1 / 2}\left(M_{2}^{-1} V\right)^{1 / 2} c^{\frac{3(q-1)}{q+3}}\left(M_{1}^{-1} U\right)^{\frac{q+1}{q+3}} k_{1}(t) .
\end{aligned}
$$

We now select $c$ such that the quantity \{\} in (3.22) vanishes. We are then led to the inequality

$$
\begin{aligned}
& U^{\prime}(t) \leq \\
& A(q) M_{1}^{\frac{q+1}{q+3}} M_{2}^{\frac{2 q}{q+3}}\left(k_{1}(t)\right)^{\frac{4 q}{q+3}}\left(M_{1}^{-1} U\right)^{\frac{q+1}{q+3}}\left(M_{2}^{-1} V\right)^{\frac{2 q}{q+3}},
\end{aligned}
$$

with

$$
A(q):=\frac{q+3}{2}(C(q))^{\frac{4 q}{q+3}}\left(\frac{3(q-1)}{4 q(2 q-1)}\right)^{\frac{3(q-1)}{q+3}} .
$$

Finally we make use of (3.10) to write

$$
\begin{aligned}
& \left(M_{1}^{-1} U\right)^{\frac{q+1}{q+3}}\left(M_{2}^{-1} V\right)^{\frac{2 q}{q+3}} \\
& =\left(\left(M_{1}^{-1} U\right)^{\frac{q+1}{3 q+1}}\left(M_{2}^{-1} V\right)^{\frac{2 q}{3 q+1}}\right)^{\frac{3 q+1}{q+3}} \\
& \leq\left\{\frac{q+1}{3 q+1} c\left(M_{1}^{-1} U\right)+\frac{2 q}{3 q+1} c^{-\frac{q+1}{2 q}}\left(M_{2}^{-1} V\right)\right\}^{\frac{3 q+1}{q+3}},
\end{aligned}
$$

and select $c$ to satisfy $(q+1) c=2 q c^{-(q+1) / 2 q}$, leading to

$$
\begin{aligned}
& \left(M_{1}^{-1} U\right)^{\frac{q+1}{q+3}}\left(M_{2}^{-1} V\right)^{\frac{2 q}{q+3}} \\
& \leq\left(\frac{q+1}{3 q+1}\right)^{\frac{3 q+1}{q+3}}\left(\frac{2 q}{q+1}\right)^{\frac{2 q}{q+3}} \Phi^{\frac{3 q+1}{q+3}} .
\end{aligned}
$$

Inserted in (3.23), we obtain

$$
M_{1}^{-1} U^{\prime}(t) \leq \Gamma(q) M_{1}^{-\frac{2}{q+3}} M_{2}^{\frac{2 q}{q+3}}\left(k_{1}(t)\right)^{\frac{4 q}{q+3}} \Phi^{\frac{3 q+1}{q+3}},
$$

with

$$
\begin{aligned}
\Gamma(q):= & \frac{q+3}{2}\left(\frac{3(q-1)}{4 q(2 q-1)}\right)^{\frac{3(q-1)}{q+3}}\left(\frac{q+1}{3 q+1}\right)^{\frac{3 q+1}{q+3}} \\
& \times\left(\frac{2 q}{q+1}\right)^{\frac{2 q}{q+3}}(C(q))^{\frac{4 q}{q+3}} .
\end{aligned}
$$

A similar computation leads to

$$
M_{2}^{-1} V^{\prime}(t) \leq \Gamma(p) M_{1}^{\frac{2 p}{p+3}} M_{2}^{-\frac{2}{p+3}}\left(k_{2}(t)\right)^{\frac{4 p}{p+3}} \Phi^{\frac{3 p+1}{p+3}} .
$$

If we suppose that

$$
\Phi(t) \rightarrow \infty \text { as } t \rightarrow t^{*},
$$

then there exists $t_{1} \geq 0$ such that $\Phi(t) \geq 1 \forall t \geq t_{1}$ and we have

$$
\Phi^{\prime}(t)=M_{1}^{-1} U^{\prime}+M_{2}^{-1} V^{\prime} \leq\left\{\begin{array}{l}
k(t) \Phi^{2 \sigma /(\sigma+1)} \text { if } \Omega \subset \mathbb{R}^{2} \\
\tilde{k}(t) \Phi^{3 \sigma /(\sigma+3)} \text { if } \Omega \subset \mathbb{R}^{3}
\end{array}\right.
$$

valid for $t \geq t_{1}$, with

$$
\begin{gathered}
\sigma:=\max \{p, q\}, \\
k(t):=F(q) M_{1}^{-\frac{1}{q+1}} M_{2}^{\frac{q}{q+1}}\left(k_{1}(t)\right)^{\frac{2 q}{q+1}} \\
+F(p) M_{1}^{\frac{p}{p+1}} M_{2}^{-\frac{1}{p+1}}\left(k_{2}(t)\right)^{\frac{2 p}{p+1}}, \\
\tilde{k}(t):=\Gamma(q) M_{1}^{-\frac{2}{q+3}} M_{2}^{\frac{2 q}{q+3}}\left(k_{1}(t)\right)^{\frac{4 q}{q+3}} \\
+\Gamma(p) M_{1}^{\frac{2 p}{p+3}} M_{2}^{-\frac{2}{p+3}}\left(k_{2}(t)\right)^{\frac{4 p}{p+3}},
\end{gathered}
$$

Integrating (3.31), we obtain in the two-dimensional case

$$
\begin{aligned}
\frac{\sigma+1}{\sigma-1} & =\int_{1}^{\infty} \Phi^{-2 \sigma /(\sigma+1)} \mathrm{d} \Phi \leq \int_{t_{1}}^{t^{*}} k(t) \mathrm{d} t \\
& \leq \int_{0}^{t^{*}} k(t) \mathrm{d} t=: K\left(t^{*}\right),
\end{aligned}
$$

from which we obtain a lower bound for $t^{*}$ of the form

$$
t^{*} \geq K^{-1}\left(\frac{\sigma+1}{\sigma-1}\right),
$$

where $K^{-1}$ is the inverse function of $K$. In the threedimensional case, we obtain

$$
\frac{\sigma+3}{2(\sigma-1)} \leq \int_{t_{1}}^{t^{*}} \tilde{k}(t) \mathrm{d} t \leq \int_{0}^{t^{*}} \tilde{k}(t) \mathrm{d} t=: \tilde{K}\left(t^{*}\right),
$$

from which we obtain a lower bound for $t^{*}$ of the form

$$
\tilde{t}^{*} \geq \tilde{K}^{-1} \frac{\sigma+3}{2(\sigma-1)} .
$$

These results are summarized in the following 


\section{Theorem 2}

Under the assumption (3.30), a lower bound for the blow-up time $t^{*}$ of the solution $(u, v)$ of $(1.1)$ is given by (3.36) in the two-dimensional case and by (3.38) in the three-dimensional case.

In the particular case in which $k_{1}(t)$ and $k_{2}(t)$ are constant, we have

$$
\begin{aligned}
t^{*} \geq & \frac{\sigma+1}{\sigma-1}\left\{F(q) M_{1}^{-\frac{1}{q+1}} M_{2}^{\frac{q}{q+1}} k_{1}^{\frac{2 q}{q+1}}\right. \\
& \left.+F(p) M_{1}^{\frac{p}{p+1}} M_{2}^{-\frac{1}{p+1}} k_{2}^{\frac{2 p}{p+1}}\right\}
\end{aligned}
$$

in the two-dimensional case and

$$
\begin{aligned}
t^{*} \geq & \frac{\sigma+3}{2(\sigma-1)}\left\{\Gamma(q) M_{1}^{-\frac{2}{q+3}} M_{2}^{\frac{2 q}{q+3}} k_{1}^{\frac{4 q}{q+3}}\right. \\
& \left.+\Gamma(p) M_{1}^{\frac{2 p}{p+3}} M_{2}^{-\frac{2}{p+3}} k_{2}^{\frac{4 p}{p+3}}\right\}
\end{aligned}
$$

in the three-dimensional case.

Theorem 2 could easily be extended to systems of $n$ parabolic equations of the form

$$
\frac{\partial u_{i}}{\partial t}=\Delta u_{i}+k_{i}(t) f_{i}\left(u_{j}\right), j \neq i=1, \cdots, n .
$$

\section{Sobolev Type Inequality in $\mathbb{R}^{2}$}

The Sobolev type inequality (3.8) in $\mathbb{R}^{2}$ may be known, but for the convenience of the reader we present a proof here.

\section{Lemma 1}

Let $u(x, y)$ be a nonnegative piecewise $C^{1}$-function defined in a bounded domain $\Omega$ that vanishes on the boundary $\partial \Omega$. Let $q$ be any constant $\geq 1$. Then we have the following Sobolev type inequality

$$
\iint_{\Omega} u^{4 q} \mathrm{~d} x \mathrm{~d} y \leq \frac{q^{2}}{2} \iint_{\Omega} u^{2(q-1)}|\nabla u|^{2} \mathrm{~d} x \mathrm{~d} y \iint_{\Omega} u^{2 q} \mathrm{~d} x \mathrm{~d} y,
$$

valid for $\Omega \subset \mathbb{R}^{2}$.

For the proof of (4.1), we follow the argument of Payne in [21]. We note that (4.1) is equivalent to

$$
\iint_{\Omega} \tilde{u}^{4 q} \mathrm{~d} x \mathrm{~d} y \leq \frac{q^{2}}{2} \iint_{\Omega} \tilde{u}^{2(q-1)}|\nabla \tilde{u}|^{2} \mathrm{~d} x \mathrm{~d} y \iint_{\Omega} \tilde{u}^{2 q} \mathrm{~d} x \mathrm{~d} y,
$$

where $\tilde{\Omega}$ is the convex hull of $\Omega$, and $\tilde{u}:=u,(x, y) \in \Omega, \tilde{u}=0,(x, y) \in \tilde{\Omega} \backslash \Omega$. It is therefore sufficient to establish (4.1) for $\Omega$ convex. For the proof, let $P:=(\bar{x}, \bar{y})$ be an arbitrary point in $\Omega \subset \mathbb{R}^{2}$. Let $P_{k}:=\left(x_{k}, \bar{y}\right) \in \partial \Omega, Q_{k}:=\left(\bar{x}, y_{k}\right) \in \partial \Omega, k=1,2$ be two pairs of boundary points associated to $P$ with $x_{1} \leq x_{2}, y_{1} \leq y_{2}$. Since $u$ vanishes on $\partial \Omega$, we have for any constant $q \geq 1$

$$
u^{2 q}(P)=2 q \int_{P_{1}}^{P} u^{2 q-1} u_{x} \mathrm{~d} x=-2 q \int_{P_{2}}^{P} u^{2 q-1} u_{x} \mathrm{~d} x,
$$

from which we obtain

$$
u^{2 q}(P) \leq q \int_{P_{1}}^{P_{2}} u^{2 q-1}\left|u_{x}\right| \mathrm{d} x
$$

Similarly we have

$$
u^{2 q}(P) \leq q \int_{Q_{1}}^{Q_{2}} u^{2 q-1}\left|u_{y}\right| \mathrm{d} y .
$$

Multiplying (4.4) by (4.5) and integrating over $\Omega$ leads to

$$
\begin{aligned}
& \iint_{\Omega} u^{4 q} \mathrm{~d} x \mathrm{~d} y \leq q^{2} \iint_{\Omega} u^{2 q-1}\left|u_{x}\right| \mathrm{d} x \mathrm{~d} y \iint_{\Omega} u^{2 q-1}\left|u_{y}\right| \mathrm{d} x \mathrm{~d} y \\
& \leq q^{2}\left(\iint_{\Omega} u^{2(q-1)} u_{x}^{2} \mathrm{~d} x \mathrm{~d} y \iint_{\Omega} u^{2(q-1)} u_{y}^{2} \mathrm{~d} x \mathrm{~d} y\right)^{1 / 2} \iint_{\Omega} u^{2 q} \mathrm{~d} x \mathrm{~d} y \\
& \leq \frac{1}{2} q^{2} \iint_{\Omega} u^{2(q-1)}|\nabla u|^{2} \mathrm{~d} x \mathrm{~d} y \iint_{\Omega} u^{2 q} \mathrm{~d} x \mathrm{~d} y,
\end{aligned}
$$

which is the desired inequality (4.1). We note that we have used the Schwarz and the arithmetic-geometric mean inequalities in the two last steps of (4.6).

\section{REFERENCES}

[1] L. E. Payne and P. W. Schaefer, "Blow-Up Phenomena for Some Nonlinear Parabolic Systems," International Journal of Pure and Applied Mathematics, Vol. 48, No. 2, 2008, pp. 193-202.

[2] L. E. Payne and G. A. Philippin, "On Blow-Up Phenomena for Solutions of a Class of Nonlinear Parabolic Problems with Time Dependent Coefficients under Dirichlet Boundary Conditions," Proceedings of the American Mathematical Sociery, accepted.

[3] V. A. Galaktionov and J. L. Vazquez, "The Problem of Blow-Up in Nonlinear Parabolic Equations," Journal of Dynamical and Control System, Vol. 8, No. 3, 2002, pp. 399-433. doi:10.1023/A:1016334621818

[4] A. A. Samarskii, V. A. Galaktionov, S. P. Kurdyumov and A. P. Mikhailov, "Blow-Up in Quasilinear Parabolic Equations," Walter de Gruyter \& Co., Berlin, 1995. doi:10.1515/9783110889864

[5] B. Straughan, "Explosive Instabilities in Mechanics," Springer, Berlin, 1998. doi:10.1007/978-3-642-58807-5

[6] C. Bandle and H. Brunner, "Blow-Up in Diffusion Equations: A Survey," Journal of Computational and Applied Mathematics, Vol. 97, No. 1-2, 1998, pp. 3-22. doi:10.1016/S0377-0427(98)00100-9

[7] L. E. Payne, G. A. Philippin and P. W. Schaefer, "Bounds for Blow-Up Time in Nonlinear Parabolic Problems," Journal of Mathematical Analysis and Applications, Vol. 338, No. 1, 2008, pp. 438-447. doi:10.1016/j.jmaa.2007.05.022

[8] L. E. Payne, G. A. Philippin and P. W. Schaefer, "BlowUp Phenomena for Some Nonlinear Parabolic Problems," Nonlinear Analysis: Theory, Methods \& Applications, Vol. 69 , No. 10, 2008, pp. 3495-3502. doi:10.1016/j.na.2007.09.035 
[9] L. E. Payne, G. A. Philippin and S. Vernier-Piro, "BlowUp Phenomena for a Semilinear Heat Equation with Nonlinear Boundary Condition, I," Zeitschrift für Angewandte Mathematik und Physik, Vol. 61, No. 6, 2010, pp. 9991007. doi:10.1007/s00033-010-0071-6

[10] L. E. Payne, G. A. Philippin and S. Vernier-Piro, "BlowUp Phenomena for a semilinear Heat Equation with Nonlinear Boundary Condition, II," Nonlinear Analysis: Theory, Methods \& Applications, Vol. 73, No. 4, 2010, pp. 971-978. doi:10.1016/j.na.2010.04.023

[11] L. E. Payne and P. W. Schaefer, "Lower Bound for BlowUp Time in Parabolic Problems under Neumann Conditions," Applicable Analysis, Vol. 85, No. 10, 2006, pp. 1301-1311. doi:10.1080/00036810600915730

[12] L. E. Payne and P. W. Schaefer, "Lower Bound for Blow -Up Time in Parabolic Problems under Dirichlet Conditions," Journal of Mathematical Analysis and Applications, Vol. 328, No. 2, 2007, pp. 1196-1205. doi:10.1016/j.jmaa.2006.06.015

[13] L. E. Payne and P. W. Schaefer, "Bounds for the BlowUp Time for the Heat Equation under Nonlinear Boundary Conditions," Proceedings of the Royal Society of Edinburgh, Vol. 139, No. 6, 2009, pp. 1289-1296.

[14] L. E. Payne and J. C. Song, "Lower Bounds for the BlowUp Time in a Temperature Dependent Navier-Stokes Flow," Journal of Mathematical Analysis and Applications, Vol. 335, No. 1, 2007, pp. 371-376. doi:10.1016/j.jmaa.2007.01.083

[15] P. Quittner, "On Global Existence and Stationary Solutions of Two Classes of Semilinear Parabolic Equations," Commemtationes Mathematicae Universitatis Carolinae, Vol. 34, No. 1, 1993, pp. 105-124.

[16] P. Quittner and P. Souplet, "Superlinear Parabolic Problems. Blow-Up, Global Existence and Steady States," Birkhäuser, Basel, 2007.

[17] J. L. Vazquez, "The Problem of Blow-Up for Nonlinear Heat Equations. Complete Blow-Up and Avalanche Formation," Rendiconti Lincei Matematica e Applicazioni, Vol. 15, No. 34, 2004, pp. 281-300.

[18] F. B. Weissler, "Local Existence and Nonexistence for Semilinear Parabolic Equations in $L^{P}$," Indiana University Mathematics Journal, Vol. 29, No. 1, 1980, pp. 79-102. doi:10.1512/iumj.1980.29.29007

[19] F. B. Weissler, "Existence and Nonexistence of Global Solutions for a Heat Equation," Israël Journal of Mathematics, Vol. 38, No. 1-2, 1981, pp. 29-40.

[20] G. Talenti, "Best Constant in Sobolev Inequality," Annali di Matematica Pura ed Applicata, Vol. 110, No. 1, 1976, pp. 353-372.

[21] L. E. Payne, "Uniqueness Criteria for Steady State Solutions of the Navier-Stokes Equations," In: Atti del Simposio Internazionale Sulle Applicazioni Dell'Analisi Alla Fisica Matematica, Cagliari-Sassari, 1964, pp. 130-153. 